Canadian

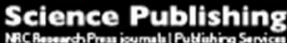

Biochemistry and Cell Biology Biochimie et biologie cellulaire

\title{
Sulforaphane Modulates Telomerase Activity via Epigenetic Regulation in Prostate Cancer Cell Lines.
}

\begin{tabular}{|r|l|}
\hline Journal: & Biochemistry and Cell Biology \\
\hline Manuscript ID & bcb-2015-0038.R1 \\
\hline Manuscript Type: & Article \\
\hline Date Submitted by the Author: & O6-Jul-2015 \\
\hline Complete List of Authors: & $\begin{array}{l}\text { Abbas, Ata; Marshall University, Biological Sciences } \\
\text { Hall, Adam; Marshall University, Biological Sciences } \\
\text { Patterson, William; Marshall University, Biological Sciences } \\
\text { Ho, Emily; Oregon State University, } \\
\text { Hsu, Anna; Oregon State University, } \\
\text { Al-Mulla, Fahd; Kuwait University, } \\
\text { Georgel, Philippe; Marshall University, Biological Sciences }\end{array}$ \\
\hline Keyword: & $\begin{array}{l}\text { Sulforaphane, Telomerase, Chromatin, Histone post-translational } \\
\text { modifications, Prostate cancer }\end{array}$ \\
\hline
\end{tabular}




\section{Sulforaphane Modulates Telomerase Activity via Epigenetic Regulation in Prostate}

\section{Cancer Cell Lines.}

Ata Abbas ${ }^{1,2, \#}$, J. Adam Hall ${ }^{1,2,3}$, William L. Patterson $3^{\text {rd 1,2,3 }}$, Emily Ho ${ }^{4}$, Anna Hsu ${ }^{4}$, Fahd Al-Mulla ${ }^{5}$, and Philippe T. Georgel ${ }^{1,2,3,6}$

1. Department of Biological Sciences, Marshall University, Huntington, WV, USA

2. Cell Differentiation and Development Center, Marshall University, Huntington, WV, USA

3. Department of Biochemistry and Microbiology, Marshall University School of Medicine, Huntington, WV, USA 4. Oregon State University, School of Biological and Population Health Sciences, Linus Pauling Institute, Corvallis, OR, USA

5. Kuwait University, Health Sciences Center, Faculty of Medicine, Molecular Pathology Unit, Kuwait City, Kuwait 6. Corresponding author \# Current address: Department of Basic Sciences, University of North Dakota School of Medicine and Health Sciences, Grand Forks, ND, USA

Running Title: SFN Epigenetically Regulates hTERT

Correspondence should be addressed to:

Philippe T. Georgel

Marshall University

Department of Biological Sciences

1 John Marshall Drive, Huntington, WV 25755

E-mail: georgel@ marshall.edu

Phone: (304) 696-3965

Fax: (304) 696-7136 


\section{Abstract}

Epidemiologic studies have revealed that diets rich in sulforaphane (SFN), an isothiocyanate present in cruciferous vegetables, are associated with a marked decrease in prostate cancer incidence. The chemo-preventive role of SFN is associated with its histone de-acetylase inhibitor activity. However, the effect of SFN on chromatin composition and dynamic folding, especially in relation to HDAC

inhibitor activity, remains poorly understood. In this study, we found that SFN can inhibit the expression and activity of human Telomerase Reverse Transcriptase (hTERT), the catalytic subunit of telomerase, in two prostate cancer cell lines. This decrease in gene expression is correlated with SFN-induced changes in chromatin structure and composition. The SFN-mediated changes in levels of histone post-translational modifications, more specifically acetylation of histone H3 Lysine 18 and di-methylation of histone H3 Lysine 4, two modifications linked with high risk of prostate cancer recurrence, were associated with regulatory elements within the hTERT promoter region. Chromatin condensation may also play a role in SFN-mediated hTERT repression; since expression and recruitment of MeCP2, a known chromatin compactor, were altered in SFN treated prostate cancer cells. Chromatin immuno-precipitation (ChIP) of MeCP2 showed enrichment over regions of the hTERT promoter with increased nucleosome density. These combined results strongly support a role for SFN in the mediation of epigenetic events leading to the repression of hTERT in prostate cancer cells. This ability of SFN to modify chromatin composition and structure associated with target gene expression provides a new model by which dietary phytochemicals may exert their chemoprevention activity.

Key words: Sulforaphane/ telomerase/ chromatin/ histone post-translational modifications/ prostate cancer/ HDAC 


\section{Introduction}

Prostate cancer (PCa) is currently the second leading cause of death in men in the United States (Adlercreutz 1990). A recent emphasis on the role of diet in cancer prevention and treatment has identified several compounds as beneficial in limiting carcinogenesis. More specifically, epidemiologic studies have linked diets rich in cruciferous vegetables, such as broccoli, Brussels sprouts, and cauliflower, with a marked reduction in PCa risk (Hebert et al. 1998; Giovannucci et al. 2003). Sulforaphane (SFN), an isothiocyanate metabolic by-product of glucoraphanin, appears to be the primary bioactive compound responsible for this effect (Bhamre et al. 2009). SFN has been shown to play an important role in inducing antioxidant electrophile response defense (phase 2) enzymes in PCa cells (Brooks et al. 2001), and is also associated with significant changes in expression of genes involved in the regulation of cell growth and cell cycle (Bhamre et al. 2009; Shan et al. 2006). Investigation of the role and effects of SFN on various types of cancer has led to the identification of several genes whose expression is affected by SFN treatment (for review, see Juge et al. 2007). SFN has been shown to inhibit prostate cancer (PCa) cell proliferation in a p53-independent manner (Fimognari et al. 2006), making it an attractive agent to treat tumors with mutated p53. This anti-proliferative activity of SFN has been correlated with increased expression of tumor suppressor and cyclin-dependent kinase inhibitor $\mathrm{p} 21^{\mathrm{CIP} 1}$. Taken together, the interpretation of these findings suggests that SFN activity may exert itself through induction of apoptosis and regulation of cell growth arrest (Chiao et al. 2002). In addition to these roles, at physiologically significant concentrations (5 to $15 \mu \mathrm{M}$ ), SFN has been shown to be involved in epigenetic regulation of

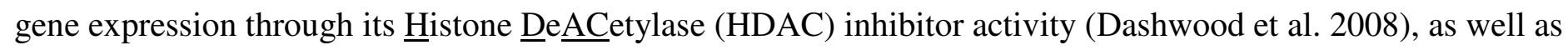
potentially affecting DNA methylation (Hsu et al. 2011). In addition, SFN-induced increase in acetylation of histones $\mathrm{H} 3$ and $\mathrm{H} 4$ has been correlated with increased expression of important genes such as $\mathrm{p} 21^{\mathrm{CIP} 1}$ and Bax (Wang et al. 2008).

The maintenance or attrition of telomeres plays an important role in cell proliferation, processes involved in differentiation, and senescence (Liu et al. 2004; Kyo et al. 2008). Loss of control in this process and re-activation of

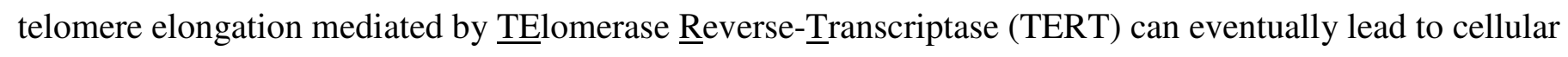
immortality and carcinogenesis in a large number of human cancers making it an important potential target for therapy. 
type-specific (Devereux et al. 1999; Guilleret et al. 2002) and may not be the most reliable marker to investigate TERT activation or repression. Histone $\mathrm{N}$-termini acetylation and methylation are also involved in transcriptional regulation of human TERT (hTERT) (Cong et al. 2000). Importantly, a recent study using several breast cancer cell lines has demonstrated that SFN represses hTERT transcriptional expression by modulating various active and inactive histone markers over the hTERT promoter region (Meeran et al. 2010). Based on this evidence and the demonstration that specific histone post-translational modifications can be used as predictors of PCa risk (Seligson et al. 2005) combined with the reported HDAC inhibitor activity of SFN we decided to investigate the interactions of various epigenetic events that can regulate hTERT expression. Our studies revealed that hTERT regulation in PCa cells is highly complex and requires, not only, cross talk between histone post-translational modifications and but was also affected by the recruitment of chromatin-associated proteins (CAP) to regulate the recruitment of specific transcription factors. In addition to these results confirming standard epigenetic modifications as important players, we provide evidence suggesting that chromatin structure (nucleosome "density" and compaction) has a significant impact on the regulation of hTERT expression.

\section{Materials and Methods}

\section{Cell culture:}

The prostate cancer cell lines LNCaP (androgen-dependent) and DU-145 (androgen-independent) were cultured (gifts from Dr. Emily Ho, Oregon State University, and Dr. Pier Paolo Claudio, Marshall University) in RPMI 1640 media (Thermo Scientific Hyclone \# 30027LS) containing $0.1 \mathrm{mg} / \mathrm{ml}$ of penicillin, $0.1 \mathrm{mg} / \mathrm{ml}$ of streptomycin, supplemented with $10 \%$ Fetal Bovine Serum at $37^{\circ} \mathrm{C}$ in $5 \% \mathrm{CO}_{2}$ incubator. Normal prostate epithelial cell line RWPE1 (gift from Dr. Pier Paolo Claudio, Marshall University) was cultured in Keratinocyte Serum Free Medium (GIBCO) supplemented with bovine pituitary extract and human recombinant epidermal growth factor. LNCaP (ATCC ${ }^{\circledR}$ CRL1740 ${ }^{\mathrm{TM}}$ ), DU-145 (ATCC ${ }^{\circledR}$ HTB-81 $1^{\mathrm{TM}}$ ) and RWPE-1 (ATCC ${ }^{\circledR}$ CRL-11609 ${ }^{\mathrm{TM}}$ ) cell lines were initially obtain from American Type Culture Collection (Manassas, VA) and were early stage of passaging at the time of experiments. The LNCaP cell line was also certified by IDDEXX RADIL ${ }^{\text {TM }}$ (Columbia, MO) on Dec 24th, 2012 to detect Mycoplasma https://mc06.manuscriptcentral.com/bcb-pubs

sp. and various STR markers. All the experiments were done within 3 months of resuscitation of cell lines. For the 
similarly to what was described by Myzak and coworkers (Myzak et al. 2006). Treatments times varied from 24 hours for gene expression analysis to up to 72 hours for the viability and cell-cycle studies.

\section{Gene Expression analysis:}

Total RNA was extracted from LNCaP and DU-145 cells cultured for 24 hours with 0 or $15 \mu \mathrm{M}$ SFN using Trizol solution (Invitrogen), and first-strand cDNA was synthesized using $1 \mu \mathrm{g}$ of total RNA and 5 units of ReverseTranscriptase. Levels of NF-kB and hTERT transcripts were quantified by quantitative Real Time PCR (qRT-PCR) and normalized to GAPDH signal. The TaqMan Gene Expression Assays (Life Technologies) were used for NF-kB (Hs00949900_m1), hTERT (Hs00972656_m1) and GAPDH (Hs99999905_m1). The PCR reaction conditions were performed as follows: $50^{\circ} \mathrm{C}$ for 2 minutes, $95^{\circ} \mathrm{C}$ for 10 minutes followed by the 40 cycles of $95^{\circ} \mathrm{C}$ for 15 seconds, and $60^{\circ} \mathrm{C}$ for one minute using an Applied Biosystems 7900HT Real-Time PCR System. The results were analysed using the ABI software.

\section{Western Blotting:}

Proteins were extracted from cultured cells using a standard RIPA lysis buffer (20 mM Tris-HCl pH 7.5, 150 $\mathrm{mM} \mathrm{NaCl}, 1 \mathrm{~m} \mathrm{M} \mathrm{Na} 2$ EDTA, $1 \mathrm{mM}$ EGTA, 1\% NP-40, 0.1\% SDS, and protease inhibitor kit (Roche)). The protein concentrations of the various samples were determined by Bradford assay (Pierce Bio-Source Kit Protein Assay) according to the manufacturer's manual. 20-40 $\mu \mathrm{g}$ of protein were electrophoresed in $8 \%$ SDS-PAGE (for proteins other than core histones) or $18 \%$ SDS-PAGE gels to monitor histone post-translational modifications. For sizing, we loaded $7 \mu$ l of Bio-Rad SDS molecular weight standards (Precision Plus Protein Standards Kaleidoscope) in the first and/or last lane of each gel. After electrophoresis, the proteins were electro-transferred to ECL nitrocellulose membranes (Amersham). Equal loading was confirmed by Ponceau staining and $\beta$-actin or GAPDH levels were used for signal normalization after stripping using Restore Western blot stripping buffer (Fisher) according to the manufacturer's directions. The membranes were blocked by incubation in PBS plus $0.05 \%$ Tween 20 and $5 \%$ non-fat milk solution. The primary antibodies (described in Supplementary Table S2) were added to fresh blocking solution https://mc06.manuscriptcentral.com/bcb-pubs

and incubated at $4^{\circ} \mathrm{C}$ overnight, followed by washes, and incubation for 1 hour with the secondary antibody. Antibody 
blots using the ECL detection system (Thermo Scientific) combined with ECL hypersensitive films. The densitometry analysis was performed using the Alpha Innotech FluorChem ${ }^{\mathrm{TM}}$ IS 9000 software.

\section{TRAP Assay:}

The telomerase activity was measured using a TRAPEZE RT Telomerase Detection Kit (EMD Millipore). The experiments were performed according to the manufacturer's instructions, monitoring addition of TTAGGG telomeric repeats. Briefly, LNCaP and DU-145 cultured for 24 hours with 0 or $15 \mu \mathrm{M}$ SFN were harvested, trypsinized, and lysed. $0.5 \mu \mathrm{g}$ of cell lysates were incubated at $30^{\circ} \mathrm{C}$ for 30 minutes, and then used to perform qRT-PCR (45 cycles: $94^{\circ} \mathrm{C}$ for 15 seconds, $59^{\circ} \mathrm{C}$ for 1 minute, then $45^{\circ} \mathrm{C}$ for 30 seconds). Quantification was performed by comparing fluorescence values from the collected samples to that generated from a standard curve obtained using a 10-fold dilution of TRS8 template (provided as control in the kit). Heat-treated samples were used as telomerase negative control. The positive control data were obtained using a TSK template (provided in the TRAPEZE RT kit). All telomerase activity measurements were performed in triplicate. The normalization of the data was performed as described in the manual of the Trapeze RT Telomerase Detection Kit protocol.

\section{Nucleosome Repeat Length (NRL) Assay:}

20-50 $\mu \mathrm{g}$ of isolated nuclei $+/-$ SFN treatment were digested with 5 units of Micrococcal Nuclease (MNase) for 1, 5, and 10 minutes at room temperature as described in Becker and $\mathrm{Wu}$ (Becker and $\mathrm{Wu}$ 1992). The digested chromatin samples were then treated with RNase 1 and digested with proteinase K (Roche), followed by organic extraction, and ethanol precipitated as described in Becker and Wu Becker and Wu 1992). Gel electrophoresis of the digested products was performed in $1 \%$ agarose in $1 \times \mathrm{TAE}$, next to a molecular weight marker containing a 100-bp ladder. After electrophoresis and ethidium bromide staining, the global average Nucleosome Repeat Lengths (NRL) were determined as described by Rodriguez-Campos and coworkers (Rodriguez-Campos et al. 1989) by measuring the distances of migration of the band corresponding to tetranucleosomes. It is important to note that repeat lengths are best evaluated using measurement from oligonucleosomes (we selected the tetranucleosome as a standard) to minimize the 
(see Supplementary S3 for primers sequences). The amplicons were labeled using the Amersham Gene Images AlkPhos Direct Labeling and Detection System according to manufacturer's specifications.

\section{Chromatin Immuno-Precipitation:}

LNCaP and DU-145 cells treated as described above ( 24 hours with 0 or $15 \mu \mathrm{M} \mathrm{SFN}$ ) were exposed to formaldehyde to a final concentration of $1 \%$ for 5 minutes at room temperature. The pelleted cells were washed and sonicated 6 times for 30 seconds at $4^{\circ} \mathrm{C}$ using a Bioruptor ${ }^{\mathrm{TM}}$ (Diagenode). The chromatin immuno-precipitations were performed using anti-bodies against H3K4me2, H3K18Ac, and MeCP2 (for details and source, see Supplementary Table S2) according to the provider's instruction. The complexes were precipitated using Dynabeads protein-G beads (Invitrogen, Cat\# 100.04D). The control ChIP was performed using IgG. The primers used for qPCR amplification are listed in Supplementary Table S3. After proper washes, the IP-ed material was incubated over-night at $65^{\circ} \mathrm{C}$ in presence of $10 \mu \mathrm{g}$ of RNase 1 and $40 \mathrm{mM} \mathrm{NaCl}$ to reverse the cross-links. The material was then ethanol precipitated and deproteinized using $10 \mu \mathrm{g}$ of proteinase K (Roche). After phenol/Chloroform extraction followed by two ethanol precipitations performed in presence of $10 \mu \mathrm{g}$ of glycogen used as carrier, the samples were air-dried and re-suspended in $30 \mu 1$ of water. The qPCR was performed in 96-well plates with Power SYBR® Green Master Mix (Life Technologies). Primers for qPCR were designed using the free open-source software Primer3 (http://frodo.wi.mit.edu/cgi-bin/primer3/primer3_www.cgi).

\section{Statistical Analysis:}

All experiments were done in triplicate and analyzed using appropriate statistical methods. One-way ANOVA was used followed by Dunnett's multiple comparison Test for the SFN concentration dependence studies. For the time dependent studies and cell-cycle analysis, we used two-way ANOVA followed by Bonferroni Post-test. The Student's t-test was used for comparing each experimental treatment value (SFN) against the corresponding control value. Standard notation was used to denote significant $\mathrm{p}$-values: ${ }^{*} \mathrm{p}<0.05,{ }^{*} \mathrm{p}<0.01,{ }^{* * *} \mathrm{p}<0.001$. 
and cytotoxic effects of SFN on RWPE-1 (normal prostate epithelial cell line; hTERT-negative), LNCaP (androgensensitive human prostate adenocarcinoma metastatic cell line; hTERT-positive) and DU-145 (androgen-insensitive prostate cancer moderately metastatic cell line; hTERT-positive) cell lines (Supplementary Figure S1). These cell lines were specifically selected to reflect the effect of SFN on normal prostate epithelial cells, androgen-sensitive, and androgen-insensitive cells. Our results indicated $15 \mu \mathrm{M}$ as an optimal concentration for our ensuing studies, since it represented the first effective dose significantly influencing cell survival, cell cycle progression, and cell death. This dose is also achievable in vivo (daily use of 100-200g of broccoli or $50 \mathrm{~g}$ of broccoli sprout has been equated to a concentration of $15 \mu \mathrm{M}$ under in vivo conditions (Heber et al. 2014, Atwell et al. 2015)), and has shown to decrease prostate tumor progression in the TRAMP mouse model for prostate cancer (Keum et al. 2009).

1. SFN affects cell proliferation, causes cell death and growth arrest in LNCaP and DU-145 cells in a time and dosedependent manner

The MTT [3-(4,5-dimethylthiazol-2-yl)-2,5-diphenyltetrazolium bromide] assay demonstrated the influence of SFN in a concentration and time-dependent manner on cell proliferation in both LNCaP and DU-145 cells (Supplementary Figure S1-B to -D). SFN, at a concentration of $15 \mu \mathrm{M}$, showed a highly significant inhibitory effect on cell proliferation in both cell lines $(\mathrm{p}<0.0001)$. The lactate dehydrogenase $(\mathrm{LDH})$ cytotoxicity assay also showed concentration- and time-dependent effects of SFN on cell death in both tested cell lines (Supplementary Figure S1-E to -F). As a control, we observed that $15 \mu \mathrm{M}$ SFN does not exert any significant effect on either cell proliferation or cell death in normal prostate epithelial cell line RWPE-1 (Supplementary Figure S1-A and -E to -F), indicating that SFN is selectively inhibiting PCa cells. The cell-cycle arrest stages after 24 hours of treatment at $15 \mu \mathrm{M}$ SFN were determined in only LNCaP and DU-145 cell lines as RWPE-1 cells were not responsive to our $15 \mu \mathrm{M}$ SFN treatment. SFN, at a concentration of $15 \mu \mathrm{M}$, blocked LNCaP cell cycle progression at the G0/G1 transition, as evidenced by an increased signal in G1 (Supplementary Figure S2-A). We also observed a significant reduction in S phase after SFN treatment in LNCaP cells. DU-145 responded to SFN treatment by showing accumulation in G2 phase (Supplementary Figure S2-

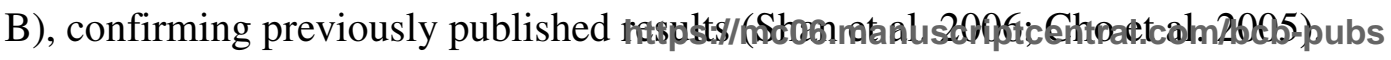


To investigate SFN's effect on telomerase transcription, we monitored mRNA levels of hTERT after 24 hours of SFN treatment $(15 \mu \mathrm{M})$ by qRT-PCR. As a control, we also monitored expression of NF- $\kappa$, which has been shown to be repressed by SFN in PC3 PCa cell (Xu et al. 2005). Our results indicated a $\sim 35$-fold repression of hTERT mRNA transcription in LNCaP, and 25-fold in DU-145 (Figure 1- A). SFN treatment caused a 20-fold decrease in NF- $\kappa \mathrm{B}$ mRNA in LNCaP, and 32-fold in DU-145 (Fig. 1-A). To confirm SFN's effect on telomerase expression at the translational level, we monitored hTERT protein level by Western blot. Our results indicated a more modest but still significant repression of hTERT in prostate cancer cell lines (Figure 1-B -C). We observed 1.8-fold decrease of hTERT protein level in LNCaP and 2.8-fold decrease in DU145 cells after SFN treatment. SFN treatment caused a significant decrease in NF- $\kappa \mathrm{B}$ expression in LNCaP, and a more modest, and yet significant, decrease in DU-145 (Figure 1-B and -C). As expected, we did not detect any hTERT expression (in the presence or absence of $15 \mu \mathrm{M}$ SFN after 24 hours) in our RWPE-1 normal prostate epithelial cell line (Figure 1-B). To confirm that the reduced expression of hTERT translated into a similar change in activity, we performed TRAP (telomeric repeat amplification protocol) assays on both cells lines. The measurement of activity was performed by quantitative RT-PCR monitoring TTAGGG extension associated with telomerase activity (Figure 1-D). A control sample, where the enzyme was heat-inactivated, was also included as a telomerase activity negative control. The results indicate that $15 \mu \mathrm{M}$ SFN treatment for 24 hours reduces hTERT activity in both LNCaP and DU-145 cells by about two and a half-fold.

\section{SFN treatment affects global epigenetic modifications}

a. DNA methylation: We first monitored the expression of DNA methyl transferases DNMT1, DNMT3a, and DNMT3b. The results revealed that the expression of all three DNMTs was down regulated in LNCaP cells (Supplementary Figure S3). In contrast, SFN treatment in DU-145 lead to up-regulation of DNMT1 and to a lesser extent DNMT3a while DNMT3b was not affected (Supplementary Figure S3). The results indicate that the two cells lines responded differently to SFN treatment. We further investigated the SFN-induced changes in DNA methylation levels over the hTERT promoter (from -201 to -7) by methylation-sensitive qPCR. This region of the promoter contains several Sp1 sites, one E2F binding site, as well as an E-box, which are known to be methylation-dependent for the https://mc06.manuscriptcentral.com/bcb-pubs

recruitment of their cognate transcription factors (Dessain et al. 2000). Our results indicated no detectable DNA 
b. Histone post-translational modifications (PTM): We monitored the effect of exposure to $15 \mu \mathrm{M}$ of SFN for 24 hours on histone N-termini acetylation in LNCaP and DU-145 cells by Western blotting. The results showed increased signals for Pan-acetylated H3 (1.9 to 4.5 fold) and Pan-acetylated H4 (7.2 to 8.2 fold) after 24 hours of SFN treatment (Figure 2), confirming the reported SFN's HDAC inhibitor activity. To further investigate lysine residue specificity for histone acetylation, and to check if histone PTMs H3K18Ac and H3K4me2 9predictors of high risk of prostate cancer recurrence (Seligson et al. 2005; 2009)) were affected, we probed several separate sets of blots with antibodies against H3K9Ac, H3K18Ac, and H3K4me2. As expected, based on SFN HDAC inhibitor activity, H3K18Ac signal was increased from 4.1 fold (LNCaP) to 4.9 fold (DU-145) after SFN treatment (Figure 2). Unexpectedly, the signal for H3K4me2 was also increased (4.3 fold in LNCaP and 11.3 fold in DU-145) by the presence of $15 \mu \mathrm{M}$ of SFN. In addition to their use as markers for actively transcribed genes (for review, see Lennartsson, et al. 2009), the combination of low histone $\mathrm{H} 3 \mathrm{~K} 18 \mathrm{Ac}$ and $\mathrm{H} 3 \mathrm{~K} 4 \mathrm{me} 2$ has been reported to be linked with the assessment of risk of PCa recurrence (Seligson et al. 2005), as well as biomarkers for lung and kidney cancers (Seligson et al. 2009). In summary, our results suggest that SFN's effect on histones may not be limited to an HDAC inhibitor role, but may also provide a histone demethylase inhibitory activity. It may also coordinate the targeting of lysines 4 and 18 on histone $\mathrm{H} 3 \mathrm{~N}-$ termini.

c. Local changes in chromatin composition and conformation: To more accurately describe the dynamic changes in location and intensity of H3K18Ac and H3K4me2 over the hTERT promoter and early transcribed region, we performed chromatin immuno-precipitation (ChIP) on LNCaP and DU-145 cells, followed by qPCR to tile the promoter region and the early transcribed region of the hTERT gene from position -3041 to +1643 . The results (Figure 3) indicate, once again, that the two cell lines behave slightly differently in response to SFN treatment. These results are very similar to what was observed by Meeran and colleagues when monitoring histones $\mathrm{H} 3$ and $\mathrm{H} 4$ pan-acetylation, H3K9Ac, H3K9me3, and H3K27me3 over the hTERT promoter region (Meeran et al. 2010). Our results showed that $\mathrm{H} 3 \mathrm{~K} 4 \mathrm{me} 2$ signal in response to SFN treatment was decreased throughout the entire region scanned in LNCaP cells (Figure 3-A). However, histone H3K18Ac signal was increased at distal region of the promoter spanning -344 to -1184 region. DU-145 cells exhibited an increase in signal for both H3K18Ac and H3K4me2 for most of the regions probed, 
The effect of SFN treatment on chromatin conformation was evaluated by measuring the global (over the entire genome) and local (over the hTERT promoter and early transcribed regions) Nucleosome Repeat Length (NRL) (Blank and Becker 1995). An increase in NRL values would suggest chromatin opening (i.e. increase in DNA accessibility, nucleosome displacement, or potential loss of histones) or presence of local nucleosome-depleted regions and would be associated with transcriptionally active regions. On the other hand, a reduced NRL would suggest chromatin "closing" or possible increased nucleosome density leading to repression of transcription. Our results indicate that, in both cell lines, the global spacing was modestly affected by SFN treatment (Figure 4). However, the local NRL over the promoter and the early hTERT transcribed region (probe 2$)$ was significantly $(\mathrm{p}<0.05)$ reduced ( 25 bp in LNCaP and 13 bp in DU-145), a result that is consistent with our reported decrease in expression (Figure 1-B). It is interesting to note that the NRL values obtained from chromatin isolated from untreated LNCaP cells were larger than their reported global counterpart. This would suggest a fairly "open" chromatin structure over the probed regions of the hTERT gene prior to SFN treatment. The SFN treatment affected the nucleosome spacing of the 5' distal promoter region (Figure 4, Probe 1) differentially in LNCaP and DU-145. LNCaP cells showed a significant decrease of 13 bp ( $\mathrm{p}<0.05)$, where DU-145 displayed no significant difference in NRL values at 5' distal promoter region. In summary, the results further suggest that SFN may epigenetically regulate hTERT transcription through related but slightly different mechanisms in hormone-responsive and -refractory prostate cancer cells.

d. Potential role of the chromatin-associated protein MeCP2 in SFN-dependent hTERT regulation of transcription: In order to further strengthen our understanding of the chromatin folding-related element, we decided to investigate the role of MeCP2, a chromatin-associated protein that has been linked with both chromatin compaction (Georgel et al. 2003), interaction with specific histone PTM (El-Osta et al. 2002), and prostate cancer cell growth (Bernard et al. 2006). Our results showed that SFN treatment significantly decreased MeCP2 expression at the protein level in LNCaP ( $\mathrm{p}<0.01$ ), but was not significantly altered in DU-145 cells (Figure 5). To complement our analysis, we performed ChIP using an anti MeCP2 antibody to monitor MeCP2 occupancy over the hTERT promoter and early transcribed region (3041 to +1643$)$. The results indicated enrichment of MeCP2, after SFN treatment, over positions -1561 to -731 and 366 to +122 in LNCaP cells, as well as positions -744 to +122 in DU-145 cells (Figure 6). These locations correspond to the promoter and early transcribed regions of the gene. Both regions are rich in transcription factor binding sites 
results suggest that hTERT reduced expression by SFN treatment may be mediated, at least in part, through an increased nucleosome density potentially mediated by MeCP2 recruitment.

\section{Discussion}

SFN affects PCa cell lines, at least in part, by blocking cell cycle progression. However, the stage of the cell cycle affected by SFN differs whether the cells are androgen-sensitive (LNCaP) or androgen-insensitive (DU-145). In contrast, the control cell, RWPE-1 did not seem to be highly affected by SFN (Figure S1-A and -D to -F). The differential effect that is reported suggests that SFN treatment may affect different sets of genes linked to cell cycle regulation in the two cell lines tested, leading to the arrest at G0/G1 or G2/M (Shan et al. 2006; Cho et al. 2005; Beaver et al. 2014). The monitoring of hTERT expression before and after SFN treatment clearly showed that its expression was significantly reduced in both PCa cell lines (note that the RWPE-1 cells were confirmed as hTERT-negative). The concomitant decrease in hTERT activity that we reported here is probably due to the observed decrease in expression, but may also be attributed to possible post-translational changes of hTERT as, for example, phosphorylation of hTERT has been shown to be involved in regulating its expression and/or SFN-induced changes in expression of other hTERTassociated proteins (Cons et al. 2002). As we attempted to pinpoint the epigenetic mechanism(s) by which SFNdependent hTERT repression is mediated, we identified a combination of specific histone PTMs (post-translational modifications) known to be involved in evaluating the risk of PCa recurrence (H3K4me2, H3K18Ac, (Seligson et al. 2005; 2009)), a chromatin-associated protein (MeCP2 (Georgel et al. 2003)), and changes in chromatin architecture as important factors in that process.

\section{1. $\underline{\text { Histone post-translational modifications }}$}

Global changes: SFN treatment induces global changes in two of the histone PTMs described as related to PCa recurrence (Seligson et al. 2005; 2009). H3K18Ac and H3K4me2 are both usually associated with actively transcribed region of the genome (Lennartsson et al. 2009), and mostly observed close to the Transcription Start Site (TSS). The observed changes in histone acetylation, including that of H3K18Ac were expected as SFN has been shown to be a 
activity. The presence of both histone PTMs, if co-localizing may be a strong determinant in the specificity of the recruitment of chromatin-associated proteins (in our case: MeCP2) involved in the regulation of hTERT transcription. Local changes: ChIP results followed by tilling indicate that the hTERT promoter region and early-transcribed regions are affected by SFN treatment in both tested PCa cell lines (DU-145 and LNCaP), but the pattern of changes is slightly different. LNCaP shows that the hTERT promoter region of SFN-treated cells has a lower incidence of H3K18Ac and H3K4me2, two markers of actively transcribed regions (with the exception of positions -1184 to -344 ). The region spanning -744 to -344 contains multiple MZF-2 binding sites. MZF-2 is a potential repressor of hTERT (Fujimoto et al. 2000) and increased H3K18Ac signals at MZF-2 binding regions might contribute to MZF-2 mediated hTERT repression in LNCaP cells. The pattern of location of decreased H3K18Ac and H3K4me2 signals is suggesting that the changes in histone PTMs are coordinated. The pattern observed with DU-145 cells seems to confirm the correlation between the two histone PTMs in response to SFN treatment. However, the increase in signal for both H3K18Ac and $\mathrm{H} 3 \mathrm{~K} 4 \mathrm{me} 2$ in presence of SFN over most of the promoter region and the early-transcribed region was not expected. It suggests that the standard correlation between these two PTMs and actively transcribed regions may not explain how hTERT is repressed in DU-145. This unexpected pattern of histone PTMs for a repressed gene has been reported in other instances. For example, recent evidence has led to a re-evaluation of the role of $\mathrm{H} 3 \mathrm{~K} 4 \mathrm{me} 2$ and $\mathrm{H} 3 \mathrm{~K} 4 \mathrm{me} 3$ in promoter activation (Pinskaya et al. 2009a, 2009b). These two histone PTMs can also be associated with a delayed or poor recruitment of RNA pol II (Pinskaya et al. 2009a, 2009b), which would result in a lower level of expression of the gene(s) affected. Several genes have also been demonstrated to have increased H3K18Ac, and yet be thoroughly repressed (Smith 2008; Barber et al. 2012; Kelly et al. 2013). Another study by Meeran and colleagues indicates that SFN increased the level of pan-H3Ac, pan-H4Ac, and H3K9Ac (active markers); whereas decreased the level of H3K9me3 and H3K27me3 (inactive markers) at hTERT promoter region (Meeran et al. 2010). This modulation of chromatin at regulatory regions of hTERT gene by SFN may facilitate the binding of various repressor proteins.

\section{Chromatin architecture}

Distribution of nucleosomes: The NRL decreases observed in response to SFN treatment in LNCaP were localized as https://mc06.manuscriptcentral.com/bcb-pubs

overlapping with hTERT promoter region and early-transcribed region. The NRL prior to SFN treatment suggests that 
distal promoter region, but is affected over the 3' promoter and early-transcribed region (Figure 4). The recruitment of a significant number of transcription factors present over the hTERT promoter is modulated by changes in chromatin composition and conformation. The changes that we observed in chromatin composition are, therefore, likely to play a role in the regulation of hTERT expression. ER's recruitment is strongly associated with the histone acetylation level (through HDAC recruitment and interaction with various co-repressors and co-activators, see Mann et al. 2011). Similarly, E2F, VDR, SP1, MZF2, and WT1 can interact with multiple chromatin-associated proteins (Rabinovich et al. 2008, Pike and Meyer 2010, Davie 2003,Toska et al. 2012) and consequently influence hTERT expression. In addition, the recruitment of several of these transcription factors (SP1, E-box binding proteins) can be affected by the level of DNA methylation, making the overall regulation of expression a highly complex process. This result, in addition to the differential histone PTMs population of the hTERT promoter appear to confirm our initial hypothesis for two slightly different modes of reducing hTERT expression in androgen-sensitive and androgen-insensitive PCa cell lines.

DNA methylation: Some changes in DNMTs expression were observed in response to SFN treatment, with LNCaP apparently more affected than DU-145 (with the exception of DNMT1), but when the level of CpG methylation was tested, no changes were detected over the promoter region of hTERT or early transcribed region before or after SFN treatment (24 hours at $15 \mu \mathrm{M})$. The topic of changes in methylation of the hTERT promoter region during transcriptional activation or repression remains controversial, as certain groups (Devereux et al. 1999; Guilleret et al. 2002; Liu et al. 2004) have indicated changes in CpG methylation status, when others (Liu et al. 2004), including us have not been able to detect any significant variation.

Role of MeCP2: To further investigate the possibility of chromatin architecture and composition in the repression of hTERT in response to SFN treatment, we consider that chromatin-associated proteins may mediate the repressive effect. MeCP2 has been reported as a highly efficient mediator of chromatin compaction (Georgel et al. 2003). The analysis of MeCP2 expression level did not provide us with a clear answer, as the two different cell types appeared to react to SFN treatment in a different manner. LNCaP showed a significant decrease in MeCP2 expression, where DU145's expression was not affected. The potential role of MeCP2 in hTERT repression was investigated by ChIP 
The recruitment of MeCP2 over these sections of the promoter and the hTERT early-transcribed region does not appear to be directly mediated by the presence of methylated $\mathrm{CpG}$, as none were detected under our experimental conditions. In this case, MeCP2 recruitment would be mediated by its ability to specifically associate with certain histone PTMs (Thambirajah et al. 2012) that can be indicative of either active (H3K4me2/3, H3K9/14Ac) or repressive (H3K9me2/3, H3K27me3). Our result suggests that MeCP2 binding was increased by the augmented presence of H3K4me2 (Regions -744 to -344 and -56 to +1643 , Figure 3-B) in DU-145, leading to heterochromatinization (as shown by decreased NRL, Figure 4; hTERT 3' Prom \& ETR). Note that recent evidence has led to a re-evaluation of the role of H3K4me2 and H3K4me3 in promoter activation (Pinskaya et al. 2009a, 2009b). These two histone PTMs can also be associated with a delayed or poor recruitment of RNA pol II (Pinskaya et al. 2009a, 2009b), which would result in a lower level of expression of the gene(s) affected. Our results are consistent with the recruitment of MeCP2 acting as a contextdependent transcriptional mediator (Thambirajah et al. 2012). The apparently conflicting results may be associated with specific cell types (telomerase-negative vs. telomerase-positive (Devereux et al. 1999; Guilleret et al. 2002 ; Liu et al. 2004) and androgen-dependent vs. androgen-insensitive.

Based on our reported results and published data, it appears that the mechanism by which SFN can repress hTERT transcription is more complex than anticipated, involving more than a simple change in histone acetylation level mediated by SFN treatment. Multiple epigenetic events (local distribution of histone PTMs, chromatin composition and structure) over the hTERT promoter and early-transcribed region are involved that will mediate the accessibility of transcription factors to their cognate binding sites. Based on the correlation between nucleosome distribution and potential folding and increased presence of specific histone PTM H3K4me2, we believe that the transcription factor and chromatin compactor MeCP2 play an important role in the SFN-mediated hTERT repression. Finally, our results suggest that the highly complex regulation of hTERT expression may vary, sometimes significantly, from cell line to cell line.

\section{Acknowledgments}

Thanks to Drs. Richard M. Niles and W. Elaine Hardman for their critical review of the manuscript. Additional thanks https://mc06.manuscriptcentral.com/bcb-pubs

to Valerie Elias for technical support in DNMT qPCR analysis and Benoit Moilnie for his input in mRNA 


\section{References}

Adlercreutz H. 1990. Western diet and Western diseases: some hormonal and biochemical mechanisms and associations. Scand. J. Clin. Lab Invest. Suppl. 201: 3-23.

Atwell LA, Hsu A, Wong CP, Stevens JF, Bella D, et al. 2015. Absorption and chemopreventive targets of sulforaphane in humans following consumption of broccoli sprouts or a myrosinase-treated broccoli sprout extract. Mol. Nutr. Food Res. 59(3): 424-433.

Barber MF, Michishita-Kioi E, Xi Y, Tasselli L, Kioi M, Moqtaderi Z, et al. 2012. SIRT7 links H3K18 deacetylation to maintenance of oncogenic transformation. Nature. 487(7405): 114-118.

Beaver LM, Buchanan A, Sokolowski EI, Riscoe AN, Wong CP, Chang JH, et al. 2014. Transcriptome analysis reveals a dynamic and differential transcriptional response to sulforaphane in normal and prostate cancer cells and suggests a role for SP1 in chemoprevention. Mol. Nutr. Food Res. 58(10). 2001-2013.

Becker PB, and Wu C. 1992. Cell-free system for assembly of transcriptionally repressed chromatin from Drosophila embryos. Mol. Cell. Biol. 12(5): 2241-2249.

Bernard D, Gil J, Dumont P, Rizzo S, Monte D, et al. 2006. The methyl-CpG-binding protein MECP2 is required for prostate cancer cell growth. Oncogene 25(9): 1358-1366.

Bhamre S, Sahoo D, Tibshirani R, Dill DL, and Brooks JD. 2009. Temporal changes in gene expression induced by sulforaphane in human prostate cancer cells. Prostate. 69(2): 181-190.

Blank TA, and Becker PB. 1995. Electrostatic mechanism of nucleosome spacing. J. Mol. Biol. 252(3): $305-313$.

Brooks JD, Paton VG, and Vidanes G. 2001. Potent induction of phase 2 enzymes in human prostate cells by sulforaphane. Cancer Epidemiol. Biomarkers Prev. 10(9): 949-954.

Chiao JW, Chung FL, Kancherla R, Ahmed T, Mittelman A, et al. 2002. Sulforaphane and its metabolite mediate growth arrest and apoptosis in human prostate cancer cells. Int. J. Oncol. 20(3): 631-636.

Cho SD, Li G, Hu H, Jiang C, Kang KS, et al. 2005. Involvement of c-Jun N-terminal kinase in G2/M arrest and caspase-mediated apoptosis induced by sulforaphane in DU145 prostate cancer cells. Nutr. Cancer. 52(2): 213-224.

Cong YS, and Bacchetti S. 2000. Histone deacetylation is involved in the transcriptional repression of hTERT in normal human cells. J. Biol. Chem. 275(46): 35665-35668.

Cong YS, Wright WE, Shay JW. 2002. Human telomerase and its regulation. Microbiol. Mol. Biol. Rev. 66(3): 407425 .

Dashwood RH, and Ho E. 2008. Dietary agents as histone deacetylase inhibitors: sulforaphane and structurally related isothiocyanates. Nutr. Rev. 66 (1): S36-38. 
region of the human telomerase reverse transcriptase (hTERT) gene. Cancer Res. 59(24): 6087-6090.

El-Osta A, Kantharidis P, Zalcberg JR, and Wolffe AP. 2002. Precipitous release of methyl-CpG binding protein 2 and histone deacetylase 1 from the methylated human multidrug resistance gene (MDR1) on activation. Mol. Cell Biol. 22(6): 1844-1857.

Fimognari C, Nusse M, Lenzi M, Sciuscio D, Cantelli-Forti G, et al. 2006. Sulforaphane increases the efficacy of doxorubicin in mouse fibroblasts characterized by p53 mutations. Mutat. Res. 601(1-2): 92-101.

Fujimoto K, Kyo S, Takakura M, Kanaya T, Kitagawa Y, et al. 2000. Identification and characterization of negative regulatory elements of the human telomerase catalytic subunit (hTERT) gene promoter: possible role of MZF-2 in transcriptional repression of hTERT. Nucleic Acids Res. 28(13): 2557-2562.

Georgel PT, Horowitz-Scherer RA, Adkins N, Woodcock CL, Wade PA, et al. 2003. Chromatin compaction by human MeCP2. Assembly of novel secondary chromatin structures in the absence of DNA methylation. J. Biol. Chem. 278(34): 32181-32188.

Giovannucci E, Rimm EB, Liu Y, Stampfer MJ, AND Willett WC. 2003. A prospective study of cruciferous vegetables and prostate cancer. Cancer Epidemiol. Biomarkers Prev. 12(12): 1403-1409.

Guilleret I, Yan P, Grange F, Braunschweig R, Bosman FT, et al. 2002. Hypermethylation of the human telomerase catalytic subunit (hTERT) gene correlates with telomerase activity. Int. J. Cancer. 101(4): 335-341.

Heber D, Li Z, Garcia-Lloret M, Wong AM, Lee TY, et al. 2014. Sulforaphane-rich broccoli sprout extract attenuates nasal allergic response to diesel exhaust particles. Food Funct. 5(1): 35-41.

Hebert JR, Hurley TG, Olendzki BC, Teas J, Ma Y, et al. 1998. Nutritional and socioeconomic factors in relation to prostate cancer mortality: a cross-national study. J. Natl. Cancer Inst. 90(21): 1637-1647.

Hsu A, Wong CP, Yu Z, Williams DE, Dashwood RH, et al. 2011. Promoter de-methylation of cyclin D2 by sulforaphane in prostate cancer cells. Clin. Epigenetics. 3: 3.

Juge N, Mithen RF, and Traka M. 2007. Molecular basis for chemoprevention by sulforaphane: a comprehensive review. Cell Mol. Life Sci. 64(9): 1105-1127.

Kelly RD, and Cowley SM. 2013. The physiological roles of histone deacetylases (HDAC) 1 and 2: complex co-stars with multiple leading parts. Biochem. Soc. Trans. 41(3): 741-749.

Keum YS, Khor TO, Lin W, Shen G, Kwon KH, et al. 2009. Pharmacokinetics and pharmacodynamics of broccoli sprouts on the suppression of prostate cancer in transgenic adenocarcinoma of mouse prostate (TRAMP) mice: implication of induction of Nrf2, HO-1 and apoptosis and the suppression of Akt-dependent kinase pathway. Pharm. Res. 26(10), 2324-2331.

Kyo S, Takakura M, Fujiwara T, and Inoue M. 2008. Understanding and exploiting hTERT promoter regulation for diagnosis and treatment of human cancers. Cancer Sci. 99(8): 1528-1538.

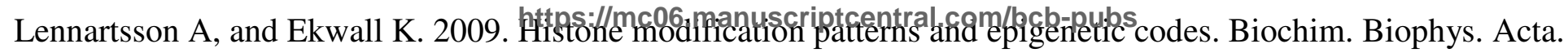
1790(9): 863-868. 
cancer progression and therapy. Cancers 3: 1691-1707.

Meeran SM, Patel SN, and Tollefsbol TO. 2010. Sulforaphane causes epigenetic repression of hTERT expression in human breast cancer cell lines. PLoS One. 5(7): e11457.

Myzak MC, Hardin K, Wang R, Dashwood RH, and Ho E. 2006. Sulforaphane inhibits histone deacetylase activity in BPH-1, LnCaP and PC-3 prostate epithelial cells. Carcinogenesis. 27(4): 811-819.

Pike JW, and Meyer MB. 2010. The Vitamin D Receptor: New paradigms for the regulation of gene expression by 1,25-dihydroxyvitamin $D_{3}$. Endocrinol. Metab. Clin. North Am. 39(2): 255-269.

Pinskaya M, Gourvennec S, and Morillon A. 2009a. H3 lysine 4 di- and tri-methylation deposited by cryptic transcription attenuates promoter activation. EMBO J. 28(2): 1697-1707.

Pinskaya M, and Morillon A. 2009b. Histone H3 lysine 4 di-methylation: a novel mark for transcriptional fidelity? Epigenetics. 4(5): 302-306.

Rabinovich A, Jin VX, Rabinovich R, Xu X, Farham P. 2008. E2F in vivo binding specificity: Comparison of consensus versus nonconsensus binding sites. Genome Res. 2008. 18(11): 1763-1777.

Rodriguez-Campos A, Shimamura A, and Worcel A. 1989. Assembly and properties of chromatin containing histone H1. J. Mol. Biol. 209(1): 135-150.

Seligson DB, Horvath S, Shi T, Yu H, Tze S, et al. 2005. Global histone modification patterns predict risk of prostate cancer recurrence. Nature. 435(7046): 1262-1266.

Seligson DB, Horvath S, McBrian MA, Mah V, Yu H, et al. 2009. Global levels of histone modifications predict prognosis in different cancers. Am. J. Pathol. 174(5): 1619-1628.

Shan Y, Sun C, Zhao X, Wu K, Cassidy A, et al. 2006. Effect of sulforaphane on cell growth, G(0)/G(1) phase cell progression and apoptosis in human bladder cancer T24 cells. Int. J. Oncol. 29(4): 883-888.

Smith CL. 2008. A Shifting paradigm: histone deacetylases and transcriptional activation. Bioessays. 30(1): 15-24.

Takakura M, Kyo S, Kanaya T, Tanaka M, and Inoue M. 1998. Expression of human telomerase subunits and correlation with telomerase activity in cervical cancer. Cancer Res. 58(7): 1558-1561.

Thambirajah AA, Ng, MK, Frehlick LJ, Serpa JJ, Petrochenko EV et al. 2012. MeCP2 binds to nucleosome free (DNA linker) regions and to H3K9/H3K27 methylated nucleosomes in the brain. Nucleic Acids Res. 40(7): $2884-2897$.

Toska E, Campbell HA, Shandilya J, Goodfellow SJ, Shore P et al. 2012. Repression of Transcription by WT1-BASP1 Requires the Myristoylation of BASP1 and the PIP2-Dependent Recruitment of Histone Deacetylase. Cell Rep. 2(3): 462-469.

Wang LG, Liu XM, Fang Y, Dai W, Chiao FB, et al. 2008. De-repression of the p21 promoter in prostate cancer cells by an isothiocyanate via inhibition of HDACs and c-Myc. Int. J. Oncol. 33(2): 375-380. 


\section{Figure Legends}

Figure1: Effect of SFN treatment $(15 \mu \mathrm{M}, 24$ hours $)$ on hTERT and NF- $\kappa$ B expression and hTERT enzymatic activity in LNCaP and DU-145 cells.

A. Alteration of hTERT and NF- $\kappa$ B mRNA expression after 24 hours $15 \mu \mathrm{M}$ SFN treatment. mRNA levels were monitored by qRTPCR. See "material and methods" section for the details. ***p<0.001.

B. The expression level of hTERT and NF- $\kappa \mathrm{B}$ in LNCaP and DU-145 cells before and after $15 \mu \mathrm{M}$ SFN treatment for 24 hours. RWPE-1 lysate was used as control that does not show hTERT expression in presence or absence of $15 \mu \mathrm{M}$ SFN treatment. The changes in expression are relative to control and were determined by densitometric scanning of each band and normalized to the $\beta$-actin control.

C. Graph representing the relative expression of hTERT and NF- $\kappa$ B using lysates from LNCaP and DU-145 cells. Each immunoblot experiment was performed in triplicate. $* * \mathrm{p}<0.01, * * * \mathrm{p}<0.001$.

D. Telomerase enzymatic activity after SFN treatment. The effect of $15 \mu \mathrm{M}$ of SFN (24 hours) on LNCaP and DU-145 cells was monitored by quantification of the TRAP products. Fold change averages (experiment performed in triplicate), after 24 hours treatment, were calculated as described in the "material and methods section". **p<0.01, $* * * \mathrm{p}<0.001$

Figure 2: Global changes in histone post-translational modifications after SFN treatment. Immunoblots for H3 Pan-acetylated (Pan Ac), H4 Pan acetylated, H3K9Ac, H3K18 Ac, and H3K4me2 were performed in triplicate using whole cell lysates. Quantification was performed after densitometry scanning and normalization to the GAPDH loading control.

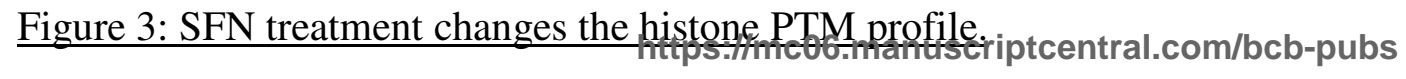

The location of H3K18Ac and H3K4me2 was assessed by ChIP followed by qPCR analysis of the precipitated DNA 
performed in triplicate. A generalized outline of the hTERT promoter and early transcribed regions is shown depicting the location of the known transcription factor binding sites (ER, -2677/-2665 and -863/-859; VDR, -2530/-2516; Sp1, 873/-868, -110/-102, -88/-83, -56/-48, -36/-28 and -7/-2; MZF-2, -687/-680, -619/-612, -543/-536 and -514/-507; WT1, -281/-273; E2F, -174/-170 and -98/-94; E-box, -165/-160 and +44/+49; ER81, +288/+291 and +390/+393). *p<0.05, $* * \mathrm{p}<0.01, * * * \mathrm{p}<0.001$

A. ChIP experiment using LNCaP cells.

B. ChIP experiment using DU-145 cells.

Figure 4: SFN treatment affects nucleosome distribution over the hTERT promoter region.

Nucleosome Repeat Length (NRL) analysis of LNCaP and DU-145 cell lines before (open bars) and after (grey bars) SFN treatment. Nuclei for the corresponding cell lines were digested with MNase, and the resulting DNA fragments electrophoresed as described in "Material and Methods". Ethidium Bromide staining revealed the global NRL (global), while Southern blotting was utilized to obtain the NRL along the 5 ' region of the hTERT promoter covering the -930 to -225 region (hTERT 5' Prom., Probe 1) and the 3' region and the Early Transcribed Region (ETR) of the same gene covering the -347 to +31 region (hTERT 3'end + ETR, Probe 2). A generalized outline of various transcription factor binding sites is shown (Sp1, -873/-868, -110/-102, -88/-83, -56/-48, -36/-28 and -7/-2; ER, -863/-859; MZF-2, -687/-680, -619/-612, -543/-536 and -514/-507; WT1, -281/-273; E2F, -174/-170 and -98/-94; E-box, -165/-160). The average NRL represents the size of the tetranucleosome fragment (in bp) divided by 4 . The data is expressed as the mean $+/$ - SE from triplicate biological samples. $* \mathrm{p}<0.05$

Figure 5: SFN treatment increases MeCP2 expression.

A. Immunoblots for MeCP2 using lysates from LNCaP and DU-145 cells before and after $15 \mu \mathrm{M}$ SFN treatment for 24 hours. The blots were stripped and re-probed using a $\beta$-actin antibody to normalize for differences in loading. https://mc06.manuscriptcentral.com/bcb-pubs

B. The relative expression levels were determined by densitometric scanning of each band and normalized to the $\beta$ - 
Figure 6: SFN treatment alters the distribution of MeCP2 over the hTERT gene promoter.

The location of MeCP2 was assessed by ChIP followed by qPCR analysis of the precipitated DNA fragments. The primers used for analysis are listed in Supplementary Table S3. The x-axis shows the location (in bp) of the amplicons. The y-axis shows the \% increase in signal observed after SFN treatment. Each experiment was performed in triplicate. A map of the hTERT promoter and early transcribed regions is shown to indicate the location of known transcription factor binding sites (ER, -2677/-2665 and -863/-859; VDR, -2530/-2516; Sp1, -873/-868, -110/-102, -88/-83, -56/-48, -36/-28 and -7/-2; MZF-2, -687/-680, -619/-612, -543/-536 and -514/-507; WT1, -281/-273; E2F, -174/-170 and -98/-94; E-box, -165/-160 and +44/+49; ER81, +288/+291 and +390/+393). ${ }^{*} \mathrm{p}<0.05, * * \mathrm{p}<0.01, * * * \mathrm{p}<0.001$.
A. ChIP experiment using LNCaP cells.
B. ChIP experiment using DU-145 cells. 
A

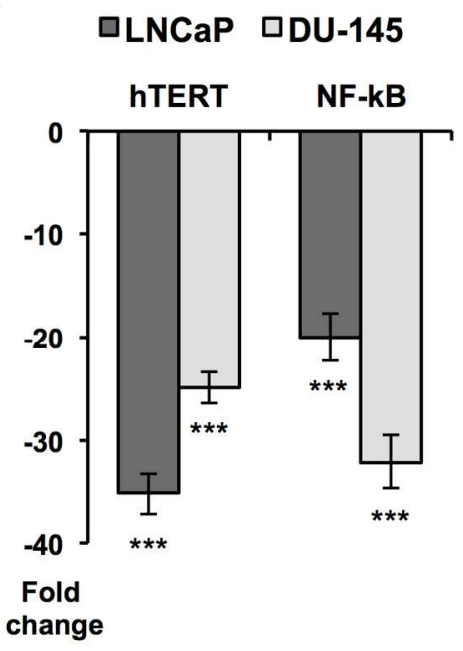

C

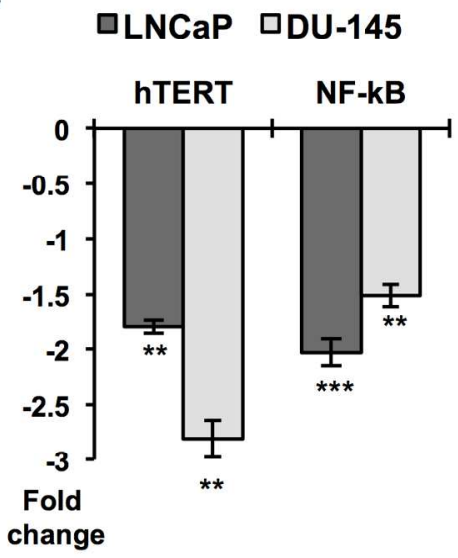

B
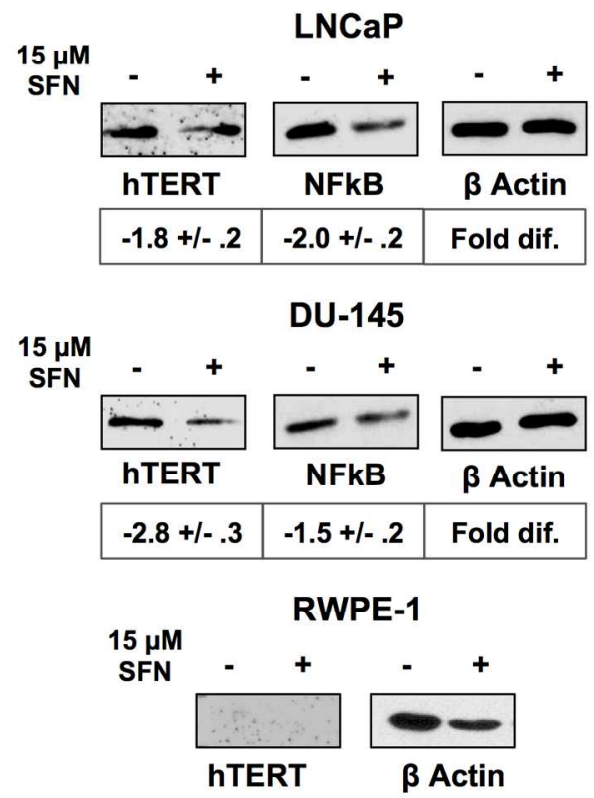

D

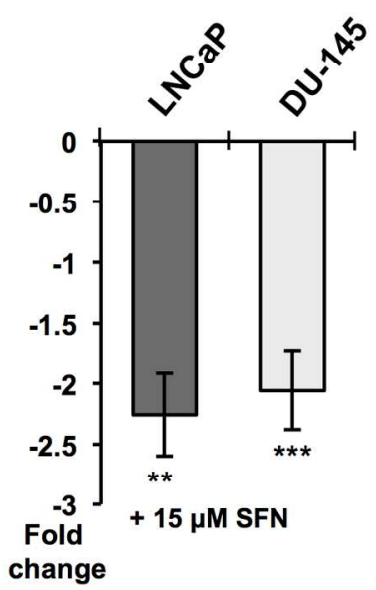

Figure1: Effect of SFN treatment (15 $\mu \mathrm{M}, 24$ hours) on hTERT and NF-KB expression and hTERT enzymatic activity in LNCaP and DU-145 cells.

A. Alteration of hTERT and NF-KB mRNA expression after 24 hours $15 \mu \mathrm{M}$ SFN treatment. mRNA levels were monitored by qRTPCR. See "material and methods" section for the details. ***p $<0.001$.

B. The expression level of hTERT and NF-KB in LNCaP and DU-145 cells before and after $15 \mu \mathrm{M} \mathrm{SFN}$ treatment for 24 hours. RWPE-1 lysate was used as control that does not show hTERT expression in presence or absence of $15 \mu \mathrm{M}$ SFN treatment. The changes in expression are relative to control and were determined by densitometric scanning of each band and normalized to the $\beta$-actin control.

C. Graph representing the relative expression of hTERT and NF-KB using lysates from LNCaP and DU-145 cells. Each immunoblot experiment was performed in triplicate. $* * p<0.01, * * * p<0.001$.

D. Telomerase enzymatic activity after SFN treatment. The effect of $15 \mu \mathrm{M}$ of SFN (24 hours) on LNCaP and DU-145 cells was monitored by quantification of the TRAP products. Fold change averages (experiment performed in triplicate), after 24 hours treatment, were calculated as described in the "material and methods section". **p $<0.01, * * * p<0.001$. 
$163 \times 190 \mathrm{~mm}(300 \times 300 \mathrm{DPI})$ 

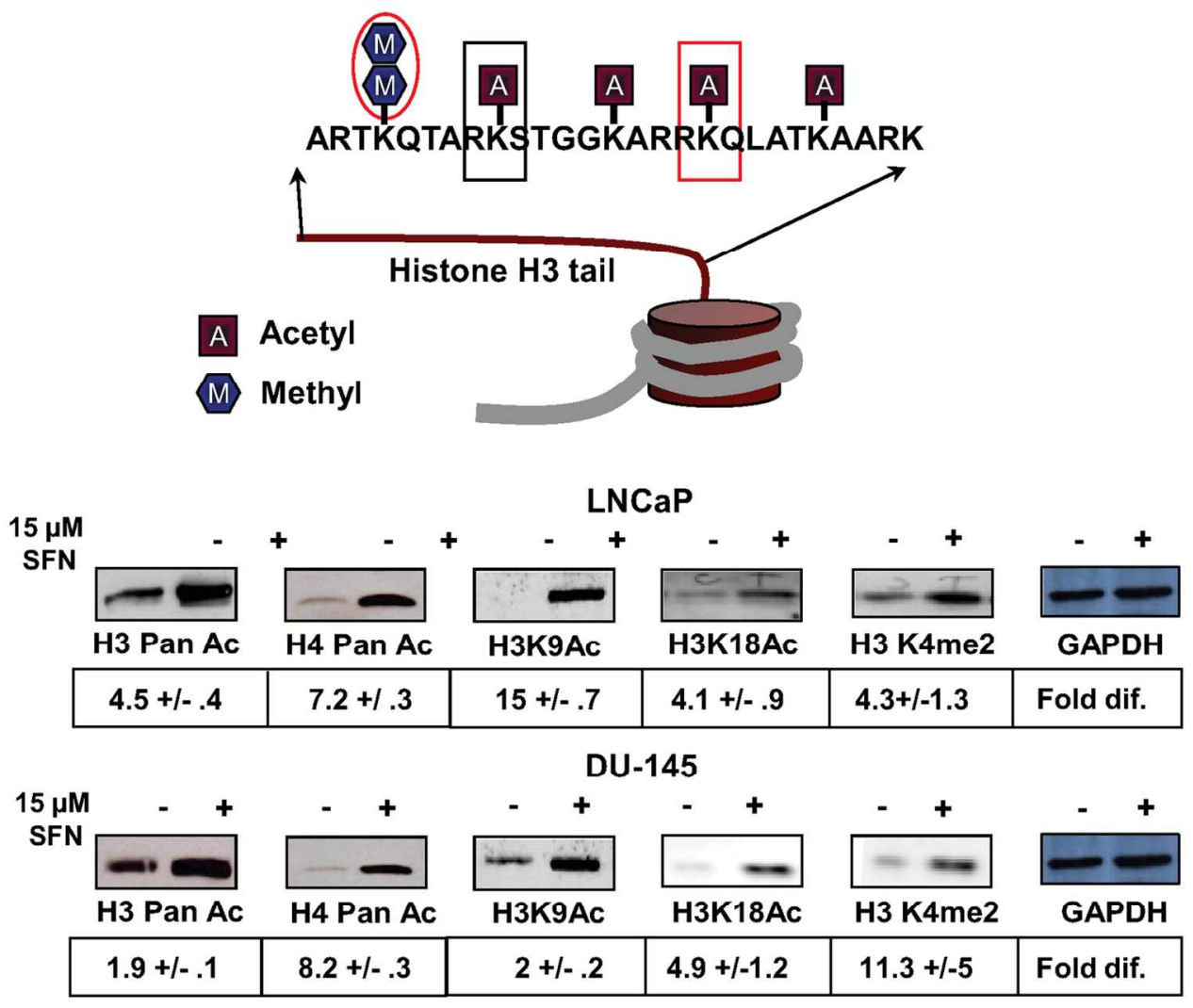

Figure 2: Global changes in histone post-translational modifications after SFN treatment. Immunoblots for H3 Pan-acetylated (Pan Ac), H4 Pan acetylated, H3K9Ac, H3K18 Ac, and H3K4me2 were performed in triplicate using whole cell lysates. Quantification was performed after densitometry scanning and normalization to the GAPDH loading control.

$129 \times 110 \mathrm{~mm}(300 \times 300 \mathrm{DPI})$ 
A

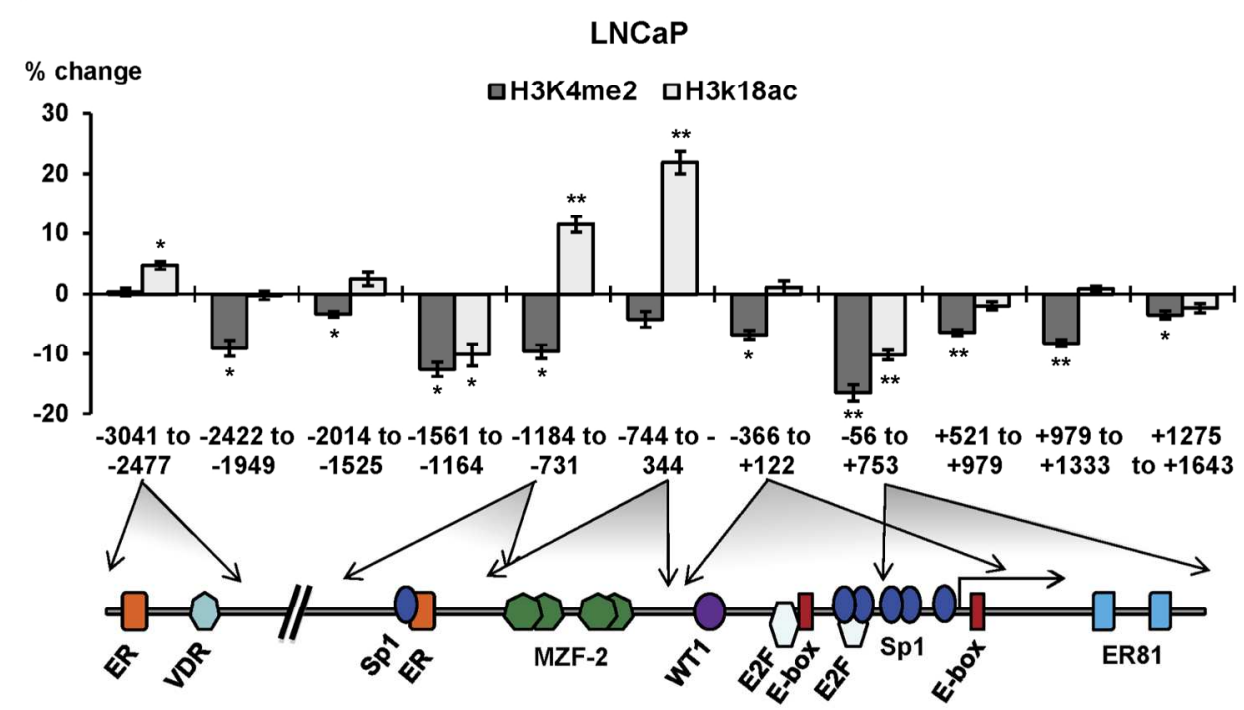

B

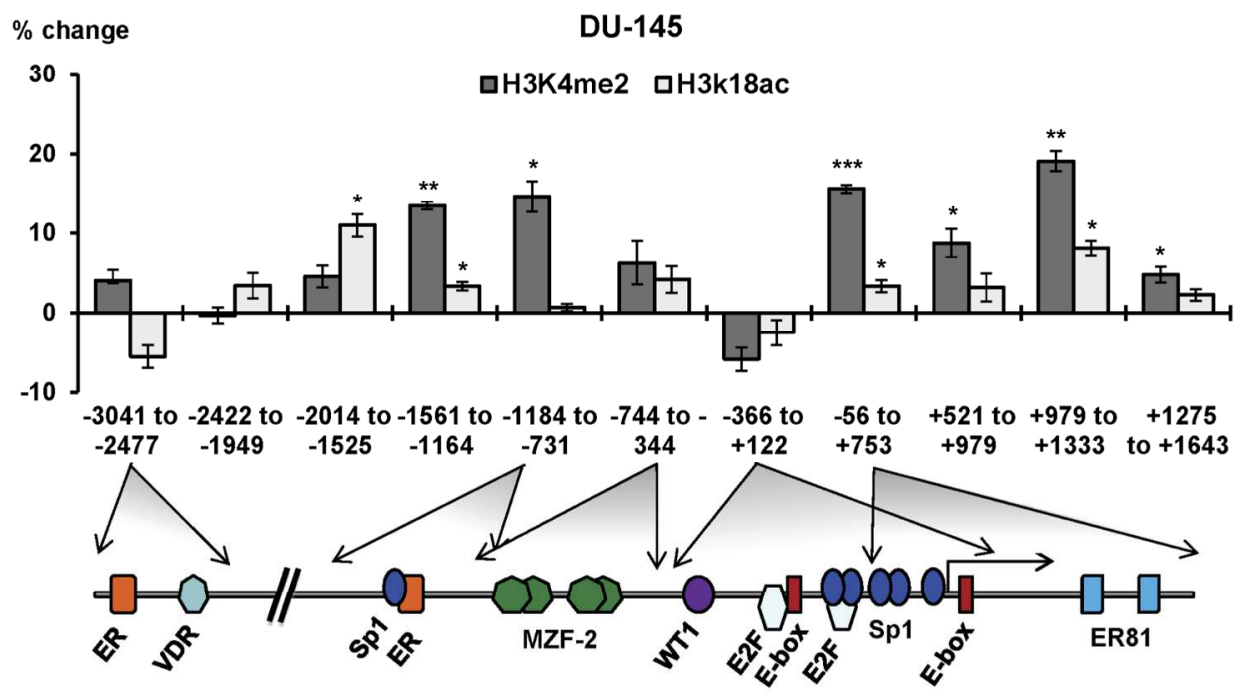

Figure 3: SFN treatment changes the histone PTM profile.

The location of H3K18AC and H3K4me2 was assessed by ChIP followed by qPCR analysis of the precipitated DNA fragments. The primers used for analysis are listed in Supplementary Table S3. The x-axis shows the location (in bp) of the amplicons. The y-axis shows the \% change in signal observed after SFN

treatment. Each experiment was performed in triplicate. A generalized outline of the hTERT promoter and early transcribed regions is shown depicting the location of the known transcription factor binding sites (ER, $-2677 /-2665$ and -863/-859; VDR, -2530/-2516; Sp1, -873/-868, -110/-102, -88/-83, -56/-48, -36/-28 and 7/-2; MZF-2, -687/-680, -619/-612, -543/-536 and -514/-507; WT1, -281/-273; E2F, -174/-170 and -98/-94; E-box, $-165 /-160$ and +44/+49; ER81, +288/+291 and +390/+393). $* p<0.05, * * p<0.01, * * * p<0.001$.

A. ChIP experiment using LNCaP cells.

B. ChIP experiment using DU-145 cells. 

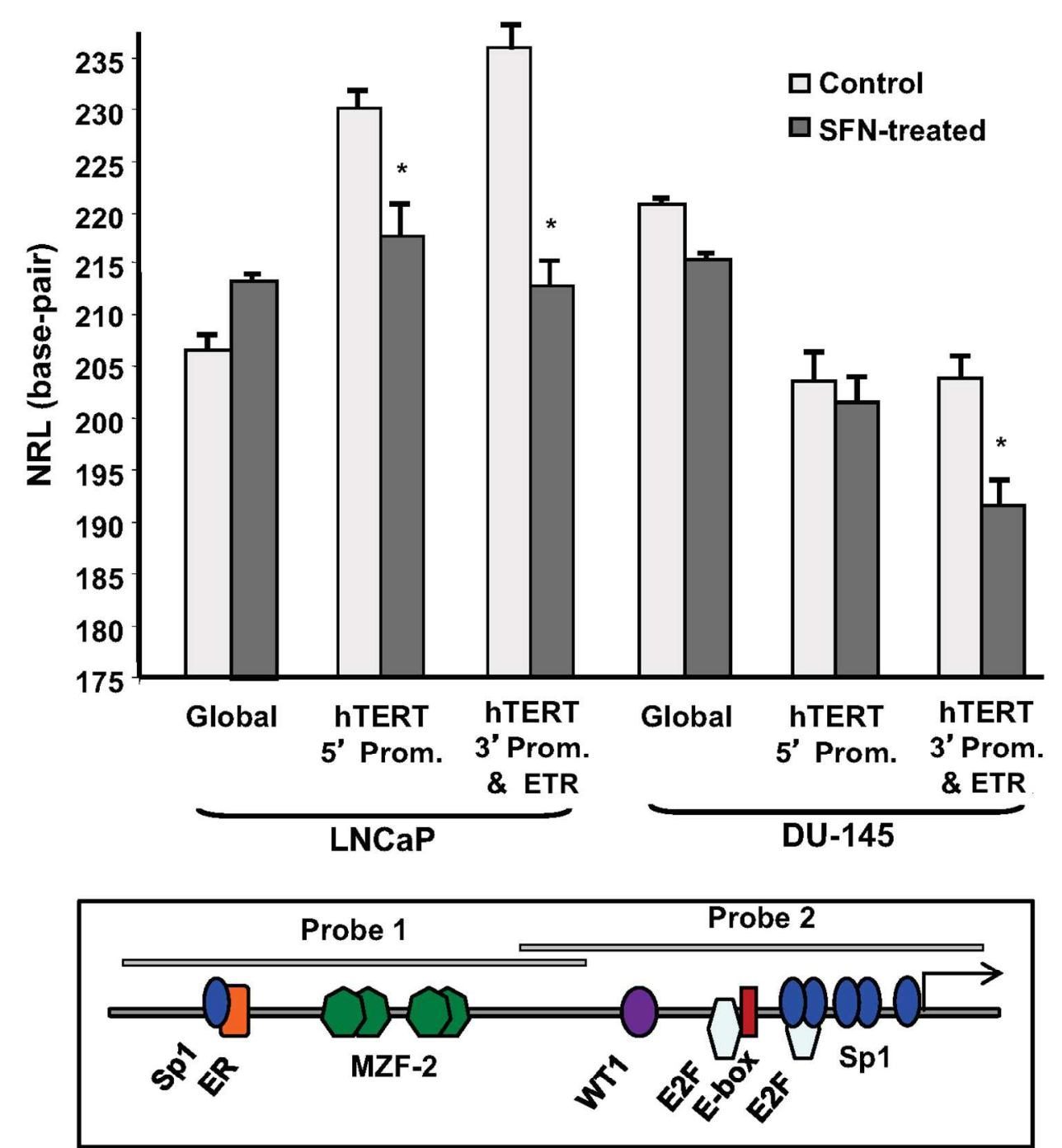

Figure 4: SFN treatment affects nucleosome distribution over the hTERT promoter region. Nucleosome Repeat Length (NRL) analysis of LNCaP and DU-145 cell lines before (open bars) and after (grey bars) SFN treatment. Nuclei for the corresponding cell lines were digested with MNase, and the resulting DNA fragments electrophoresed as described in "Material and Methods". Ethidium Bromide staining revealed the global NRL (global), while Southern blotting was utilized to obtain the NRL along the 5' region of the hTERT promoter covering the -930 to -225 region (hTERT 5' Prom., Probe 1 ) and the $3^{\prime}$ region and the Early Transcribed Region (ETR) of the same gene covering the -347 to +31 region (hTERT 3'end + ETR, Probe 2). A generalized outline of various transcription factor binding sites is shown (Sp1, $-873 /-868$, $-110 /-102,-88 /-83,-56 /-48,-36 /-28$ and -7/-2; ER, -863/-859; MZF-2, -687/-680, -619/-612, -543/-536 and 514/-507; WT1, -281/-273; E2F, -174/-170 and -98/-94; E-box, -165/-160). The average NRL represents the size of the tetranucleosome fragment (in bp) divided by 4 . The data is expressed as the mean $+/-$ SE from triplicate biological samples. * $\mathrm{p}<0.05$ 
A
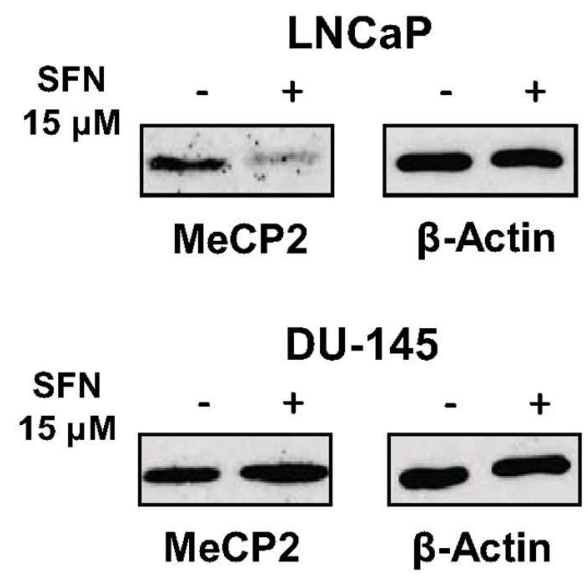

B

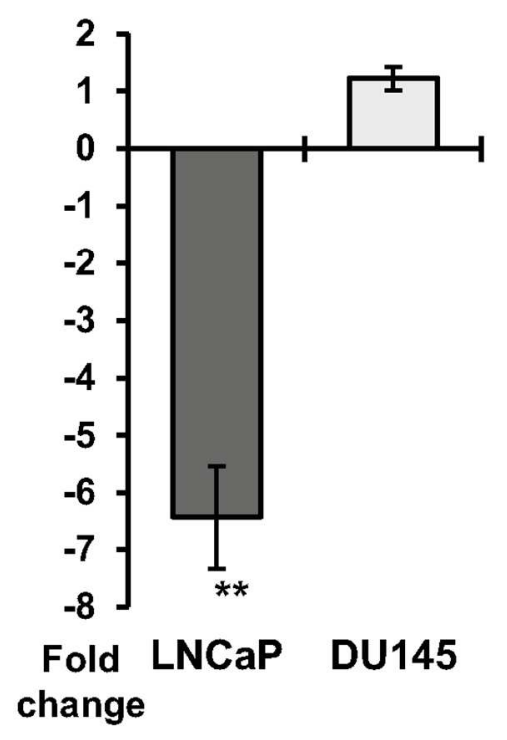

Figure 5: SFN treatment increases MeCP2 expression.

A. Immunoblots for MeCP2 using lysates from LNCaP and DU-145 cells before and after $15 \mu \mathrm{M}$ SFN treatment for 24 hours. The blots were stripped and re-probed using a $\beta$-actin antibody to normalize for differences in loading.

B. The relative expression levels were determined by densitometric scanning of each band and normalized to the $\beta$-actin control. Each immunoblot experiment was performed in triplicate. $* * p<0.01$

$63 \times 147 \mathrm{~mm}(300 \times 300 \mathrm{DPI})$ 


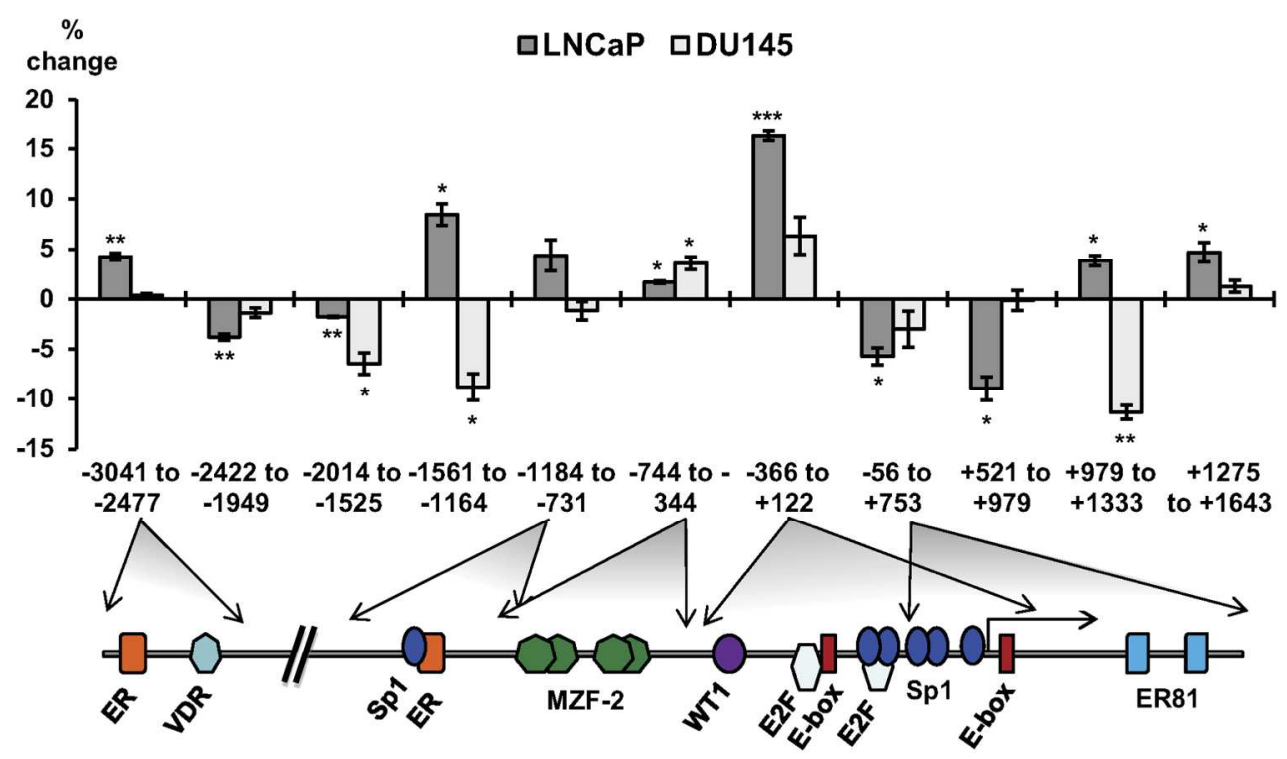

Figure 6: SFN treatment alters the distribution of MeCP2 over the hTERT gene promoter.

The location of MeCP2 was assessed by ChIP followed by qPCR analysis of the precipitated DNA fragments. The primers used for analysis are listed in Supplementary Table S3. The $x$-axis shows the location (in bp) of the amplicons. The $y$-axis shows the \% increase in signal observed after SFN treatment. Each experiment was performed in triplicate. A map of the hTERT promoter and early transcribed regions is shown to indicate the location of known transcription factor binding sites (ER, -2677/-2665 and -863/-859; VDR, -2530/2516; Sp1, -873/-868, -110/-102, -88/-83, -56/-48, -36/-28 and -7/-2; MZF-2, -687/-680, -619/-612, -543/536 and -514/-507; WT1, -281/-273; E2F, -174/-170 and -98/-94; E-box, -165/-160 and +44/+49; ER81, $+288 /+291$ and $+390 /+393) . * p<0.05, * * p<0.01, * * * p<0.001$

A. ChIP experiment using LNCaP cells.

B. ChIP experiment using DU-145 cells.

$172 \times 103 \mathrm{~mm}(300 \times 300 \mathrm{DPI})$ 